\title{
Establishing microbial baselines to identify indicators of coral reef health
}
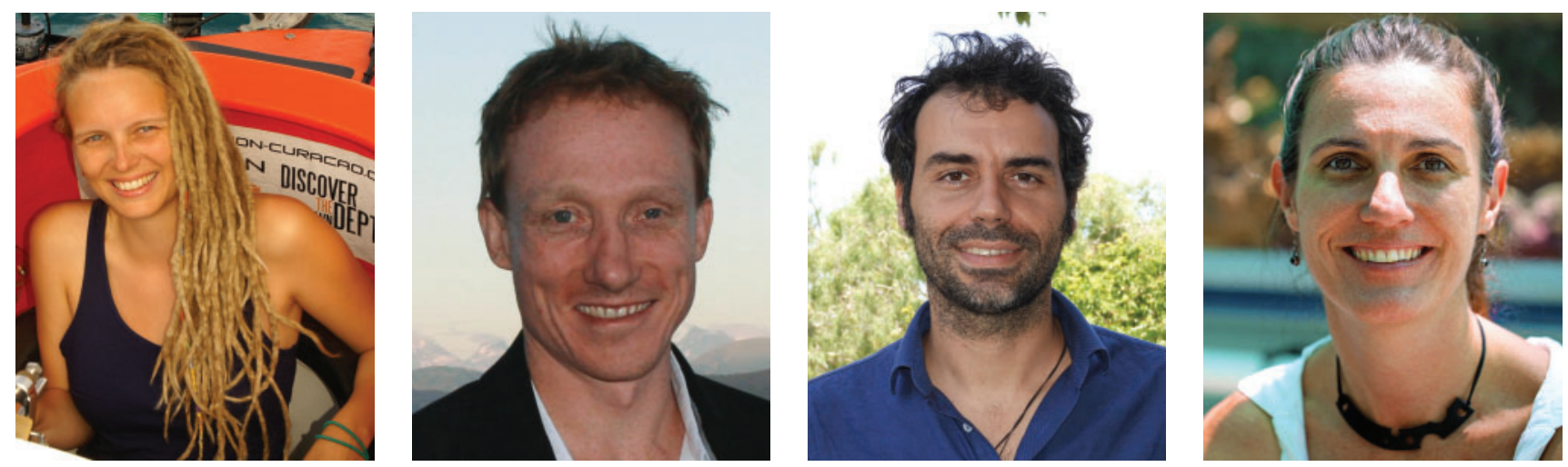

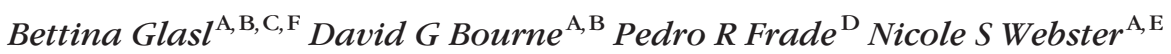

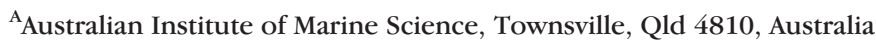

${ }^{\mathrm{B}}$ College of Science and Engineering, James Cook University, Townsville, Q1d 4811, Australia

CAIMS@JCU, Townsville, Q1d 4811, Australia

${ }^{\mathrm{D}}$ Centre of Marine Sciences, University of Algarve, 8005-139 Faro, Portugal

${ }^{\mathrm{E}}$ Australian Centre for Ecogenomics, University of Queensland, Brisbane, Qld 4072, Australia

Fel: +6174753 4444, Fax: +6174772 5852, Email: b.glasl@aims.gov.au

Microorganisms make a significant contribution to reef ecosystem health and resilience via their critical role in mediating nutrient transformations, their interactions with macro-organisms and their provision of chemical cues that underpin the recruitment of diverse reef taxa. However, environmental changes often cause compositional and functional shifts in microbial communities that can have flow-on consequences for microbial-mediated processes. These microbial alterations may impact the health of specific host organisms and can have repercussions for the functioning of entire coral ecosystems. Assessing changes in reef microbial communities should therefore provide an early indicator of ecosystem impacts and would underpin the development of diagnostic tools that could help forecast shifts in coral reef health under different environmental states. Monitoring, management and active restoration efforts have recently intensified and diversified in response to global declines in coral reef health. Here we propose that regular monitoring of coral reef microorganisms could provide a rapid and sensitive platform for identifying declining ecosystem health that can complement existing management frameworks. By summarising the most common threats to coral reefs, with a particular focus on the Great Barrier Reef, and elaborating on the role of microbes in coral reef health and ecosystem stability, we highlight the diagnostic applicability of microbes in reef management programs. Fundamental to this objective is the establishment of microbial baselines for Australia's coral reefs.

Coral reefs represent one of the most diverse ecosystems on the planet, providing home for an estimated $25 \%$ of all known marine species ${ }^{1}$. Each year Australia's iconic Great Barrier Reef (GBR) attracts millions of tourists from all over the world and provides $\$ 6.4$ billion dollars to the Australian economy ${ }^{2}$. However, reefs globally are facing unprecedented pressures ${ }^{3}$. During the past three decades, the GBR has also been severely impacted by the combined effects of climate change, crown of thorns starfish outbreaks, coral disease, overfishing and declining water quality ${ }^{3-5}$. Back-to-back bleaching events were experienced in 2016 and 2017 on the GBR, resulting in over $80 \%$ mortality of corals in some regions and an estimated loss of $29 \%$ of corals across the GBR system $^{3,6}$. In addition to global pressures related to climate change, coral reefs are also affected at local scales ${ }^{7}$. For example, the GBR is locally affected by the run-off from 35 river basins, draining an area of over $424000 \mathrm{~km}^{28}$. Intensified agricultural land use in the GBR catchment area has caused an increase of sediments, nutrients and pesticides associated with terrestrial runoff, resulting in a 
significant decline in water quality, which poses ongoing chronic and periodic acute threats to the health of the GBR?

Coral reef monitoring and management initiatives are well-established in Australia. For example, since the early 1980s the Australian Institute of Marine Science (AIMS) has assessed the health of Australia's coral reefs via its Long-Term Monitoring Program (LTMP). The Great Barrier Reef Marine Park Authority (GBRMPA) has managed the GBR area for over 40 years under the Great Barrier Reef Marine Park Act 1975. In 2015, the Australian and Queensland governments released the Reef 2050 Long-Term Sustainability Plan, outlining concrete measures to manage and protect the GBR over the next three decades. However, despite the focus on coral reef monitoring and management initiatives across all levels of government and strong community engagement in many areas, the coral reefs surrounding much of the Australian coastline, like other parts of the world, have demonstrated concerning declines in recent years ${ }^{3,5}$. One aspect that is poorly understood yet fundamental to coral reef functioning and ecosystem resilience is the contribution of microorganisms. Here we highlight that incorporating microbial based monitoring approaches into coral reef management initiatives will increase our understanding of reef ecosystem health and inform potential options for increasing reef resilience (Figure 1)

\section{Importance of microbes in coral reefs}

Microorganisms play an essential role in coral reef ecosystem processes and form diverse symbiotic relationships with benthos-dominating macro-organisms such as corals, sponges and algae $^{10-12}$ (Figure 2). The functional role of microbes in coral reefs include biochemical cycling of nutrients, degradation and remineralisation, host nutrition, vitamin synthesis, production of secondary metabolites and host defence via the production of antimicrobial peptides ${ }^{10,11}$. Microbes often form specific and stable associations with their host species ${ }^{13}$ and can assist them to acclimate to the prevailing environmental conditions ${ }^{14,15}$.

Environmental variations, such as seasonal run-off or anthropogenic-induced fluctuations in water quality are known to alter the composition and function of the reef microbiome ${ }^{16,17}$. Numerous studies have shown a clear shift in microbial community composition and function in coral reef waters and associated with dominant benthic life forms (such as corals) as the health of the ecosystem declines $^{18,19}$ (Figure 3). However, despite the recognised influence microbes have on coral reef health ${ }^{10,20}$, a holistic understanding of their dynamics in coral reef ecosystems remains elusive $^{21}$. Establishing microbial baselines that characterise the temporal and spatial microbial dynamics in coral reefs is urgently needed to underpin rapid and sensitive assessments of declining

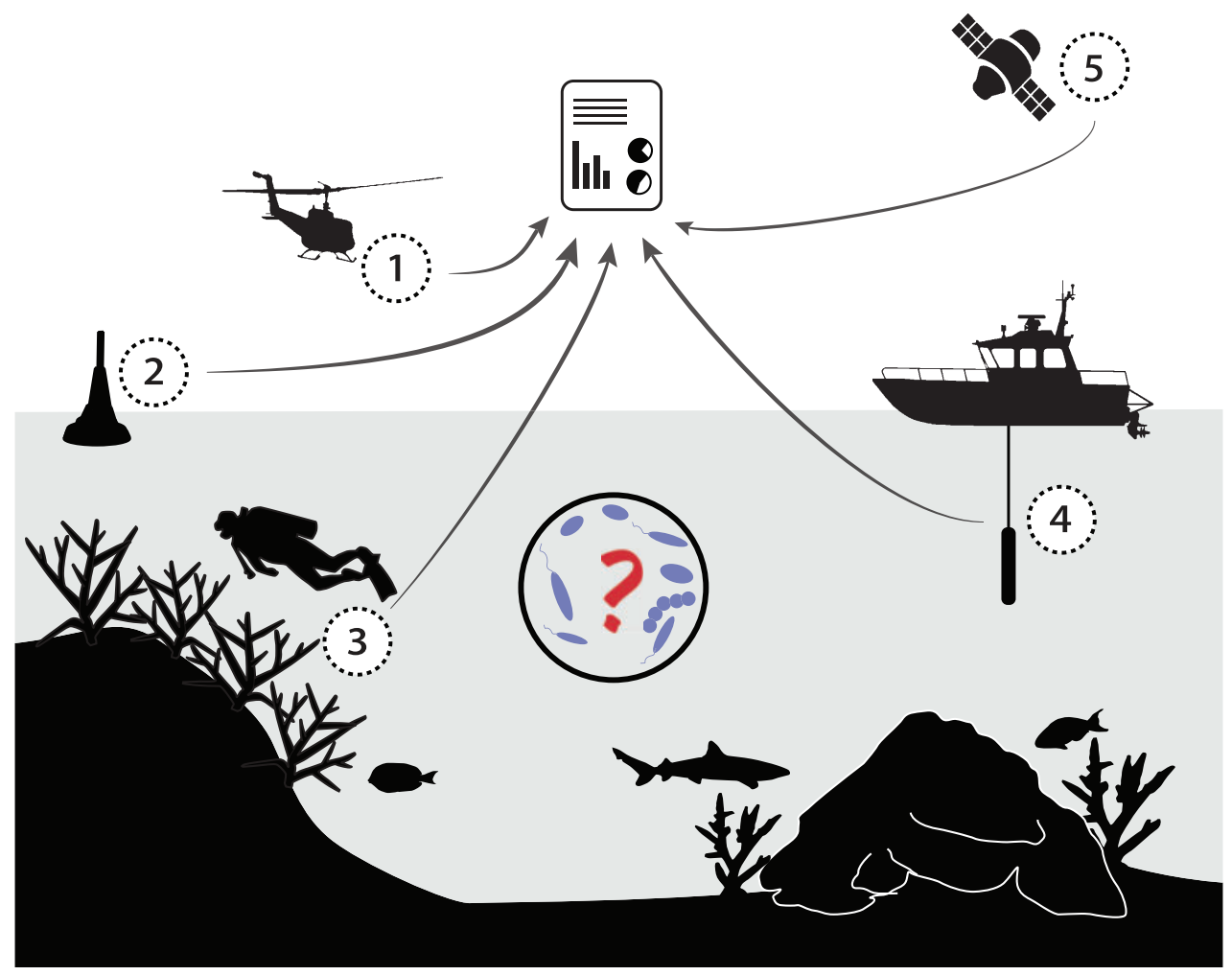

Figure 1. Implemented management strategies under Australia's Reef 2050 Long-Term Sustainability Plan are guided by an integrated monitoring approach including 1) large scale aerial surveys, 2) mooring systems and weather stations that provide data on surface (e.g. wind, precipitation, barometric pressure, temperature) and subsurface conditions (e.g. temperature, conductivity, chlorophyll fluorescence, turbidity, oxygen, light transmission and photosynthetically active radiation), 3) assessment of coral cover, coral recruitment, coral community composition and coral-macroalgae ratios on a reef, 4) comprehensive water quality assessments and screening for pesticide concentrations and 5) near surface concentration measurements of chlorophyll $a$ and total suspended solids based on remote sensing technologies. Currently, this integrated monitoring framework lacks a microbial approach and hence, excludes a considerable part of the coral reef biodiversity. 


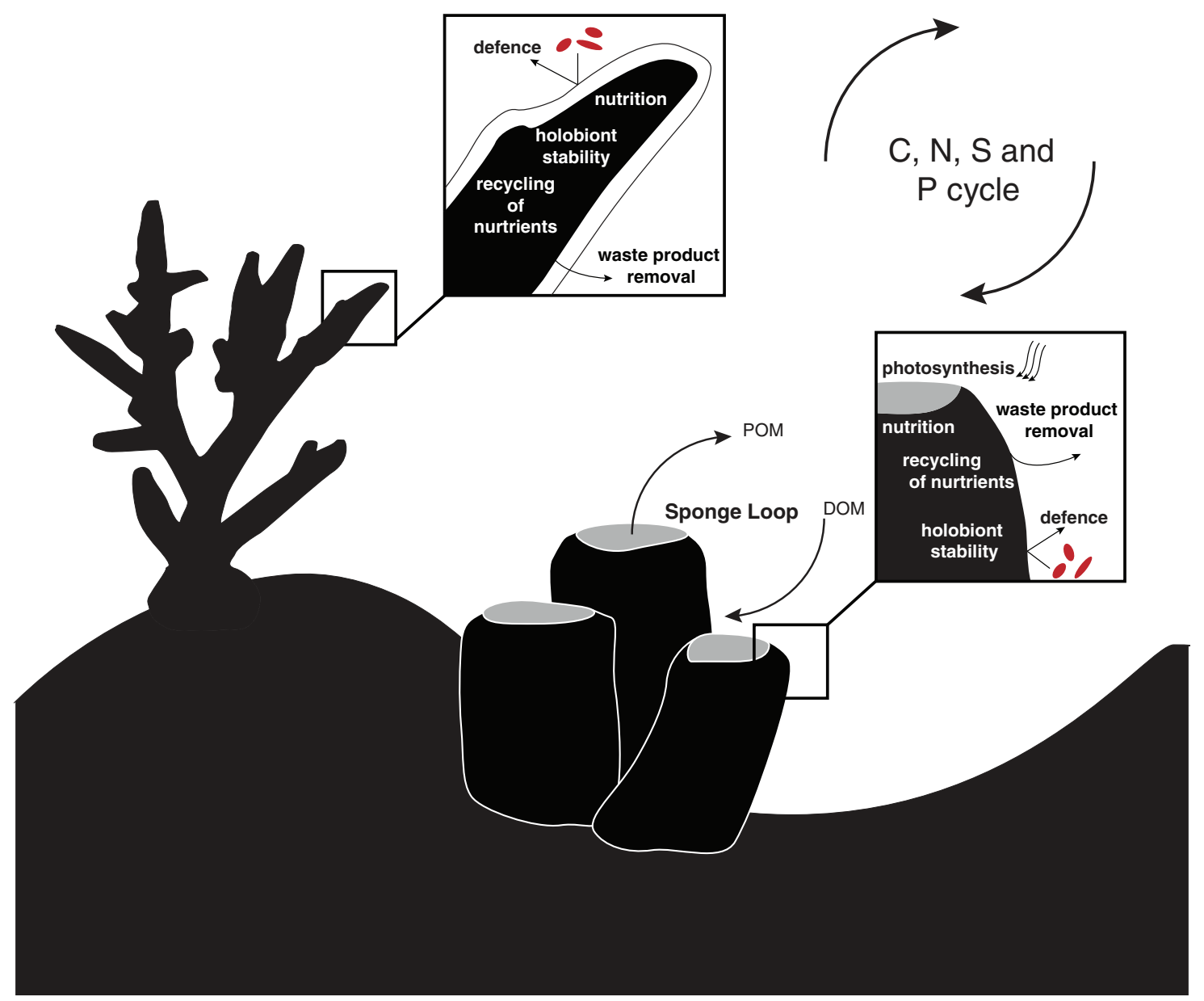

Figure 2. Simplified overview of microbial functions in a coral reef ecosystem. Microbes play a fundamental role in all major biogeochemical cycles (Carbon, Nitrogen, Sulfur and Phosphorus) in the coral reef ecosystem and contribute to their host's nutrition, waste product removal, pathogen defence and holobiont stability.

reef health and make predictions about the consequences of future environmental changes ${ }^{10,22}$

\section{Australia's initiatives to establish microbial baselines}

Recent advances in next generation sequencing technologies combined with an increased recognition of the crucial ecosystem roles played by microorganisms, has resulted in a heightened commitment to understand spatial and temporal microbial dynamics in Australian ecosystems. For example, the BASE project (Biomes of Australian Soil Environments) is the first Australian soil microbial diversity database, providing amplicon sequencing data alongside contextual data for more than 900 sites across Australia ${ }^{23}$. Another example is the Australian Marine Microbial Biodiversity Initiative (AMMBI), which was the first standardised microbial ocean observatory program undertaken at a continental scale. AMMBI aims to provide long-term microbial sequencing data from seven different pelagic sites around Australia, providing important baseline data on microbial composition and function in Australian off-shore waters. This is linked to extensive physicochemical and oceanography data derived from the Integrated Marine Observing System
(IMOS) reference stations (www.imos.org.au), allowing both hindcasting and forecasting of microbial responses to environmental conditions. Recently the Marine Microbes (MM) project (www.bioplatforms.com/marine-microbes/) was established as part of the larger AMMBI initiative to sample microbial communities associated with corals, sponges, seaweeds, seagrasses, seawater and sediment from benthic sites across Australia, including sampling locations in the GBR, Perth and Sydney. The MM project aims to provide the first holistic microbial baseline for coral reefs in Australia.

\section{Microbes as indicators of coral reef health}

Indicator organisms are used to effectively monitor habitat conditions and environmental changes ${ }^{24}$. Biological indicators are a wellestablished monitoring tool for estuarine and freshwater ecosystems $^{25,26}$ and also find application in coral reef ecosystems ${ }^{27}$. In the context of public health, microorganisms are extensively used as indicators to monitor drinking water supplies and the quality of recreational waters in order to prevent gastrointestinal illnesses $^{28,29}$. Furthermore, recent advances in human microbiome research have led to an increase of microbial based diagnostic and 


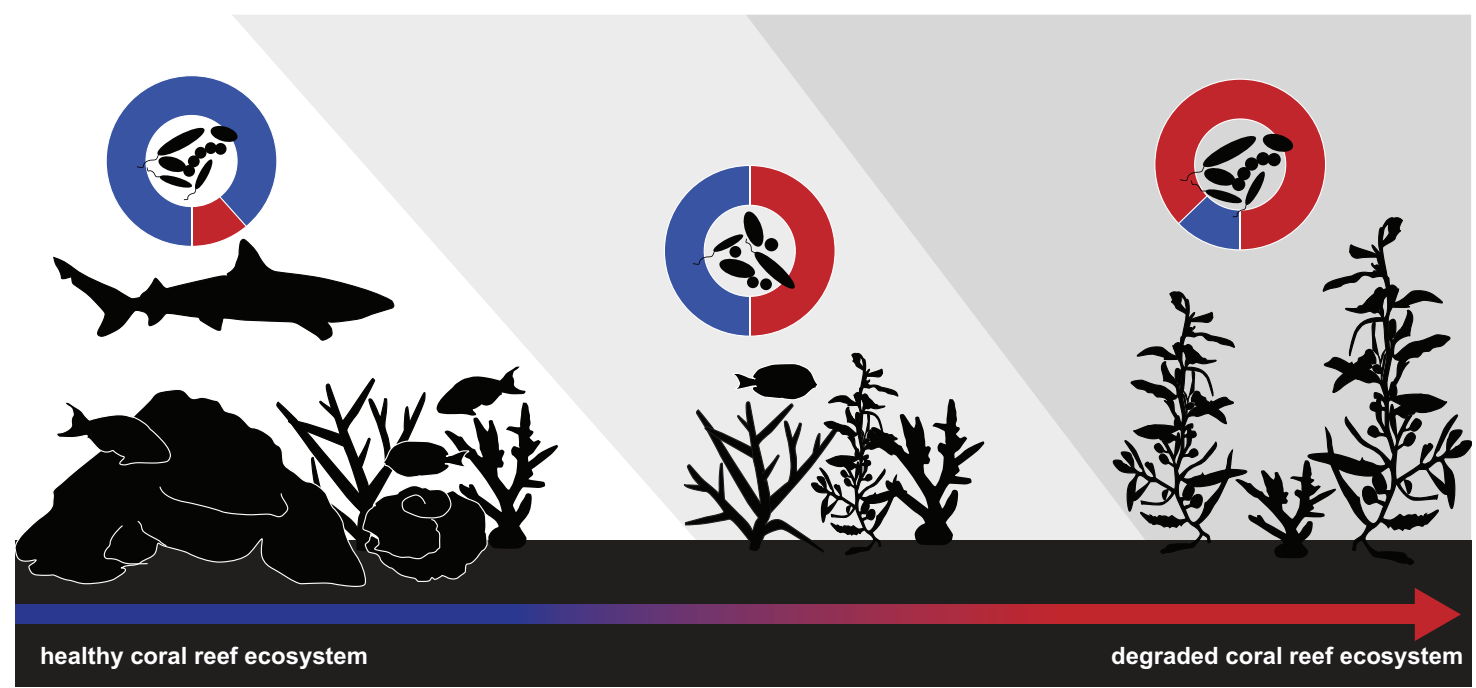

Figure 3. Coral reef ecosystems are increasingly affected by the intensification of environmental pressures emerging from land-use changes, overfishing, crown-of-thorns starfish outbreaks, coral diseases and climate change. Degradation of coral reefs and a shift from coral to macroalgae dominated benthic communities (from left to right) has been observed globally. As the health of the coral reef ecosystem changes, microorganisms rapidly respond. The microbiome of healthy reefs is dominated by beneficial and symbiotic microbes (blue), but as ecosystem health declines the microbiome shifts to an unbeneficial community, dominated by pathogens and opportunists (red).

therapeutic approaches ${ }^{30}$. Despite the emerging predictive power of the microbiome in human disease diagnostics ${ }^{30,31}$, the use of microorganisms as sensitive indicators of environmental stress in coral reef ecosystems or as predictive markers for water quality in marine systems has remained relatively unexplored ${ }^{10,22}$. Microbialisation scores are among the few attempts to monitor coral reef ecosystem declines based on the metabolic rates of microbial communities and reef-associated fishes ${ }^{32}$. Incorporating microbial monitoring tools into current coral reef health assessment programs will confer significant advantages as microbes are known to rapidly respond to changes in their environment, allowing for early diagnosis of changing water conditions and host physiological states.

Despite many potential advantages, microbial systems for monitoring coral reefs are still very much in their infancy and considerable additional research and validation would be required before microbial based monitoring approaches could be applied. Additional technical considerations that remain to be addressed include: (1) How frequently should sampling occur? (2) How and what should be sampled (e.g. seawater, sediment, microbiomes of benthic organisms such as corals or seaweed)? (3) What types of samples and analyses would be necessary (e.g. community profiling, targeted screening for particular microbial indicator taxa and/ or functions)? and (4) How to minimise costs and increase efficiency of a microbial based monitoring system to ensure real-time assessment of reef health?

\section{Conclusion}

The important role of microbes in coral reef ecosystem functioning and their contribution to the resistance and resilience of coral reefs has become widely accepted ${ }^{20,22}$. However, although Australia is at the forefront of coral reef studies and coral reef monitoring operations, to date, microbes have not been considered in large-scale monitoring approaches. The past few years have seen increased interest in understanding microbial dynamics in Australia's ecosystems, which has led to holistic sampling efforts to establish the first microbial baselines for soils and marine environments. We argue that the establishment and ongoing assessment of such microbial baselines will be crucial to understanding microbial dynamics in response to broad ranging anthropogenic impacts. The inclusion of microbial monitoring approaches alongside our current coral reef monitoring framework will improve our ability to rapidly detect changes occurring in Australian coral reefs resulting in improved protection and management of these ecologically and economically unique ecosystems.

\section{Acknowledgements}

We thank Megan Huggett for her feedback and scientific input. Funding was provided through the AIMS@JCU PhD Scholarship, GBRMPA Science Management Research Award and the Advance Queensland PhD Scholarship to BG. PRF was supported by the Portuguese Science and Technology Foundation (FCT) through fellowship SFRH/BPD/110285/2015.

\section{References}

1. Connell, J.H. (1978) Diversity in tropical rain forests and coral reefs. Science 199, 1302-1310. doi:10.1126/science.199.4335.1302

2. Deloitte. (2017) At what price? The economic, social and icon calue of the Great Barrier Reef. Deloitte Access Economics.

3. Hughes, T.P. et al. (2017) Coral reefs in the Anthropocene. Nature 546, 82-90 doi:10.1038/nature22901

4. Hoegh-Guldberg O. (2011) The impact of climate change on coral reef ecosystems. In Coral Reefs: An Ecosystem in Transition. pp. 391-403. 
5. De'ath, G. et al. (2012) The 27-year decline of coral cover on the Great Barrier Reef and its causes. Proc. Natl. Acad. Sci. USA 109, 17995-17999. doi:10.1073/ pnas.1208909109

6. Great Barrier Reef Marine Park Authority. (2017) Final report: 2016 coral bleaching event on the Great Barrier Reef. GBRMPA, Townsville.

7. Knowlton, N. and Jackson, J.B.C. (2008) Shifting baselines, local impacts, and global change on coral reefs. PLoS Biol. 6, e54. doi:10.1371/journal.pbio. 0060054

8. Brodie, J.E. et al. (2012) Terrestrial pollutant runoff to the Great Barrier Reef: an update of issues, priorities and management responses. Mar. Pollut. Bull. 65 , 81-100. doi:10.1016/j.marpolbul.2011.12.012

9. Waterhouse, J. et al. (2012) Quantifying the sources of pollutants in the Great Barrier Reef catchments and the relative risk to reef ecosystems. Mar. Pollut. Bull. 65, 394-406. doi:10.1016/j.marpolbul.2011.09.031

10. Bourne, D.G. et al. (2016) Insights into the coral microbiome: underpinning the health and resilience of reef ecosystems. Ann. Rev. Microbiol. 70, 317-340. doi:10.1146/annurev-micro-102215-095440

11. Webster, N.S. and Thomas, T. (2016) The sponge hologenome. mBio 7, e0013516. doi:10.1128/mBio.00135-16

12. Egan, S. et al. (2013) The seaweed holobiont: understanding seaweed-bacteria interactions. FEMS Microbiol. Rev. 37, 462-476. doi:10.1111/1574-6976.12011

13. McFall-Ngai, M. et al. (2013) Animals in a bacterial world, a new imperative for the life sciences. Proc. Natl. Acad. Sci. USA 110, 3229-3236. doi:10.1073/pnas. 1218525110

14. Ziegler, M. et al. (2017) Bacterial community dynamics are linked to patterns of coral heat tolerance. Nat. Commun. 8, 14213. doi:10.1038/ncomms14213

15. Webster, N.S. and Reusch, T.B.H. (2017) Microbial contributions to the persistence of coral reefs. ISME J. 11, 2167-2174. doi:10.1038/ismej.2017.66

16. Angly, F.E. et al. (2016) Marine microbial communities of the Great Barrier Reef lagoon are influenced by riverine floodwaters and seasonal weather events. Peerf 4, e1511. doi:10.7717/peerj.1511

17. Ziegler, M. et al. (2016) Coral microbial community dynamics in response to anthropogenic impacts near a major city in the central Red Sea. Mar. Pollut. Bull. 105, 629-640. doi:10.1016/j.marpolbul.2015.12.045

18. Haas, A.F. et al. (2016) Global microbialization of coral reefs. Nat. Microbiol. 1, 16042. doi:10.1038/nmicrobiol.2016.42

19. Zaneveld, J.R. et al. (2016) Overfishing and nutrient pollution interact with temperature to disrupt coral reefs down to microbial scales. Nat. Commun. $\mathbf{7}$, 11833. doi:10.1038/ncomms11833

20. Ainsworth, T.D. and Gates, R.D. (2016) Corals' microbial sentinels. Science 352, 1518-1519. doi:10.1126/science.aad9957

21. Garren, M. and Azam, F. (2012) New directions in coral reef microbial ecology. Environ. Microbiol. 14, 833-844. doi:10.1111/j.1462-2920.2011.02597.x

22. Glasl, B. et al. (2017) Microbial indicators as a diagnostic tool for assessing water quality and climate stress in coral reef ecosystems. Mar. Biol. 164, 91. doi:10.1007/s00227-017-3097-x

23. Bissett, A. et al. (2016) Introducing BASE: the Biomes of Australian Soil Environments soil microbial diversity database. Gigascience 5, 21. doi:10.1186/s13742016-0126-5

24. De Cáceres, M. et al. (2010) Improving indicator species analysis by combining groups of sites. Oikos 119, 1674-1684. doi:10.1111/j.1600-0706.2010.18334.x

25. Smith, M.J. et al. (1999) AusRivAS: using macroinvertebrates to assess ecological condition of rivers in Western Australia. Freshw. Biol. 41, 269-282. doi:10.1046/ j.1365-2427.1999.00430.x

26. Wright, J.F. (1995) Development and use of a system for predicting the macroinvertebrate fauna in flowing waters. Aust. J. Ecol. 20, 181-197. doi:10.1111/ j.1442-9993.1995.tb00531.x

27. Cooper, T.F.et al. (2009) Bioindicators of changes in water quality on coral reefs: review and recommendations for monitoring programmes. Coral Reefs $\mathbf{2 8}$, 589-606. doi:10.1007//500338-009-0512-x

28. Gruber, J.S. et al. (2014) Coliform bacteria as indicators of diarrheal risk in household drinking water: systematic review and meta-analysis. PLoS One 9, e107429. doi:10.1371/journal.pone.0107429
29. Soller, J.A. et al. (2014) Human health risk implications of multiple sources of faecal indicator bacteria in a recreational waterbody. Water Res. 66, 254-264. doi:10.1016/j.watres.2014.08.026

30. Zmora, N. et al. (2016) Taking it personally: personalized utilization of the human microbiome in health and disease. Cell Host Microbe 19, 12-20. doi:10.1016 j.chom.2015.12.016

31. Knights, D. et al. (2011) Human-associated microbial signatures: examining their predictive value. Cell Host Microbe 10, 292-296. doi:10.1016/j.chom. 2011.09.003

32. McDole, T. et al. (2012) Assessing coral reefs on a pacific-wide scale using the microbialization score. PLoS One 7, e43233. doi:10.1371/journal.pone.0043233

\section{Biographies}

Bettina Glasl is currently undertaking her PhD research at James Cook University in collaboration with the Australian Institute of Marine Science. Bettina has recently been awarded a prestigious Advance QLD PhD Scholarship by the Queensland Government. Her PhD research focuses on the temporal dynamics of coral reef microbiomes and the resistance and resilience of coral and sponge microbiomes upon environmental disturbances.

David G Bourne is a Senior Lecturer at James Cook University and a Principal Research Scientist at the Australian Institute of Marine Science (AIMS). His training is in the area of molecular microbial ecology with his research focused on investigation of microbial diversity, structure and function in complex ecosystems. His research is divided essentially into two areas, the first investigating the normal microbial communities associated with corals and their functional roles in maintaining coral fitness. The second research focus is to elucidate pathogens and mechanism of disease onset in corals and the implications this has on a stressed reef ecosystem in light of climate change being a major driver of coral reef degradation.

Pedro $\mathbf{R}$ Frade is a postdoctoral researcher at the Centre for Marine Sciences (CCMAR) at the University of Algarve (Portugal). His research focuses on the microbial ecology of coral reef ecosystems, in particular the functional role of coral-associated microbial communities belonging to the three domains of Life and their contribution to niche diversification and adaptation of corals Pedro uses field and experimental approaches to investigate the microbiome's functional diversity in auto- vs heterotrophic corals, across shallow vs mesophotic reefs, and for tropical vs temperate coral hosts

Nicole $\mathbf{S}$ Webster is a principal research scientist at the Australian Institute of Marine Science where she undertakes research into how microorganisms contribute to reef ecosystem health. Nicole also holds a joint appointment as Principal Research Fellow at the Australian Centre for Ecogenomics at the University of Queensland. In both positions Nicole uses experimental and field-based ecological research to explore multiple facets of coral reef microbiology and symbiosis. 